\title{
A Comparative Study of Full Navier-Stokes and Reduced Navier-Stokes Analyses for Separating Flows Within a Diffusing Inlet S-Duct
}

B.H. Anderson, D.R. Reddy, and K. Kapoor Lewis Research Center

Cleveland, Ohio

Prepared for the

29th AIAA Joint Propulsion Conference and Exhibit cosponsored by the AIAA, SAE, ASME, and ASEE Monterey, California, June 28-30, 1993 
A COMPARATIVE STUDY OF FULL NAVIER-STOKES AND REDUCED NAYIER-STOKES

ANALYSES FOR SEPARATING FLOWS WITHIN A DIFFUSING INLET S-DUCT

\author{
B.H. Anderson, D.R. Reddy, and K. Kapoor \\ National Aeronautics and Space Administration \\ Lewis Research Center \\ Cleveland, Ohio 44135
}

\title{
SUMMARY
}

A three-dimensional implicit Full Navier-Stokes (FNS) analysis and a 3D Reduced Navier Stokes (RNS) initial value space marching solution technique has been applied to a class of separated flow problems within a diffusing S-duct configuration characterized as vortex-liftoff. Both Full Navier-Stokes and Reduced Navier-Stokes solution techniques were able to capture to overall flow physics of vortex lift-off, however more consideration must be given to the development of turbulence models for the prediction of the locations of separation and reattachment. This accounts for some of the discrepancies in the prediction of the relevant inlet distortion descriptors, particularly circumferential distortion. The 3D RNS solution technique adequately described the topological structure of flow separation associated with vortex lift-off.

\section{NOMENCLATURE}

\begin{tabular}{|c|c|c|}
\hline$A_{i}$ & $=$ & inlet throat area \\
\hline$C_{f}$ & $=$ & wall skin friction coefficient \\
\hline$D_{i}$ & $=$ & inlet throat diameter \\
\hline$D C_{60}$ & $=$ & $\begin{array}{l}\text { distortion descriptor defined as the } \\
\text { nsaximum }\left(P t_{\text {ave }}-P t_{\min }\right) / q_{\text {ave }} \text { in any } 60.0^{\circ} \text { sector }\end{array}$ \\
\hline$L$ & $=$ & length of inlet duct \\
\hline$M_{i}$ & $=$ & inlet throat Mach number \\
\hline$P t_{0}$ & $=$ & free stream total pressure \\
\hline$P t_{\text {ave }}$ & $=$ & average total pressure at the engine face \\
\hline$P t_{\min }$ & $=$ & $\begin{array}{l}\text { minimum total pressure at engine face } \\
\text { in any sector of extent } 60.0^{\circ}\end{array}$ \\
\hline$q_{\text {ave }}$ & $=$ & average dynamic pressure at the engine face \\
\hline$R_{i}$ & $=$ & inlet throat radius \\
\hline$R_{e f}$ & $=$ & engine face radius \\
\hline Rey & $=$ & Reynolds number based on throat diameter \\
\hline & $=$ & free stream total temperature \\
\hline$X, Y, Z$ & $=$ & primary cartesian coordinates \\
\hline$X_{c l}, Y_{c l,} Z_{c l}$ & $=$ & cartesian coordinates along inlet centerline \\
\hline & $=$ & boundary layer thickness \\
\hline$\rho$ & $=$ & fluid density \\
\hline
\end{tabular}




\section{INTRODUCTION}

Modern tactical aircraft are required to be maneuverable at subsonic, transonic, and supersonic speeds, without giving up good cruise performance. Consequently; proper integration of the engine inlet with the airframe is of paramount importance.. Regarding the enhancement of inlet performance and operation, design for optimum airframe-inlet integration has the following goals: (1) to minimize approach flow angularity with respect to the inlet cowl lip, (2) to deliver uniform, high pressure recovery flow to the inlet face, (3) to prevent or minimize vortex, wake, and boundary layer ingestion by the inlet throughout the flight envelope, (4) to reduce FOD/hot gas ingestion by the inlet, and finally (5) to minimize the potential for flow field interference from weapon carriage/firing, landing gear deployment, tanks, pods, or other hardware. The combination of inlet design and airframe integration must not only provide high pressure recovery to maintain the desired thrust levels, but also generate low flow distortion consistent with stable engine operation.

Engine face flow distortion is one of the most troublesome and least understood problems for designers of modern inlet engine systems. ${ }^{1-2}$ One issue is that there are numerous sources of flow field distortion that are ingested by the inlet or generated within the inlet duct itself. Among these sources are (1) flow separation at the cowl lip during maneuvering flight, (2) flow separation on the compression surfaces due to shock-wave boundary layer interactions, (3) spillage of the fuselage boundary layer into the inlet duct, (4) ingestion of aircraft vortices and wakes emanating from upstream disturbances, and (5) secondary flow and possibly flow separation within the inlet duct itself. Most aircraft have experienced one or more of these types of problems during development, particularly at high Mach numbers and/or extreme maneuver conditions, such that flow distortion at the engine face exceeded allowable surge limits. Such compatibility problems were encountered in the early versions of the B70, the F-111, the F-14, the MIG-25, the Tornado and the Airbus A300 to name a few examples.

Flow separation in diffusing S-duct geometries characterized as vortex lift-off has been studied by Harlof, Reichert, and Wellborn ${ }^{3}$ using FNS analysis techniques and by Anderson and Farokhi ${ }^{4}$ using an RNS approach. This class of separated flows are very common within inlet ducts, and are a major cause of pressure loss and distortion at the engine face, particularly circumferential distortion. Reduced Navier-Stokes solution technique using FLARE approximations ${ }^{4}$ has been shown to physically describe the topological and topographical structure flow separation associated with vortex liftoff, however no detailed comparative study has been made between FNS and RNS viscous analyses for this phenomena over a wide range of inlet flow conditions.

The overall objective of this study is to advance the understanding, the prediction, and the control of inlet distortion, and to study the basic interactions that are involved in the management of engine face distortion within inlet ducts using Computational Fluid Dynamics. This paper examines the phenomena of vortex lift-off using both Full Navier-Stokes and Reduced Navier-Stokes solution techniques, each with an algebric eddy viscosity turbulence model. Specifically, the goals of the present paper are: (1) to examine the capability of the Full Navier-Stokes and Reduced Navier Stokes analyses to describe the phenomena of vortex lift-off over a wide range of inlet flow conditions, (2) to charactrerize the phenomena of vortex lift-off and identify uncertainties in the analysis 
of this interaction, and (3) to examine the prediction of the relevant inlet performance parameters of total pressure recovery and various engine face discriptors relative to experimental measurements.

\section{THEORETICAL BACKGROUND}

\section{Reduced Navier-Stokes Analysis}

Three dimensional viscous subsonic flows in complex inlet duct geometries are investigated by a numerical procedure which allows solution by spatial forward marching integration, utilizing flow approximations from the velocity-decomposition approach of Briley and McDonald. ${ }^{5-6}$ The goal of this approach is to achieve a level of approximation that will yield accurate flow predictions, while reducing the labor below that needed to solve the full Navier Stokes equations. The governing equations for this approach have been given previously for orthogonal coordinates, and the approach has been applied successfully to problems whose geometries can be fitted conveniently with orthogonal coordinate systems. However, geometries encountered in typical subsonic inlet ducts cannot be treated easily using orthogonal coordinates, and this led to an extension of this ap: proach by Levy, Briley, and McDonald, ${ }^{7}$ to treat ducted geometries with nonorthogonal coordinates. In generalizing the geometry formulation, Anderson, 8 extended the analysis to cover ducted geometries defined by an externally generated gridfile, such that it allowed for (1) reclustering the existing gridfile, (2) redefining the centerline space curve, and (3) altering the cross-sectional shape and area distribution without returning to the original gridfile. This version of the 3D RNS computer code is called RNS3D. The turbulence model used in RNS3D is that of McDonald and Camarata ${ }^{9}$ which employes an eddy-viscosity formulation for the Reynolds stresses.

\section{Full Navier-Stokes Analysis}

The PARC3D 10 code, selected for this study, solves the full three-dimensional, Reynolds averaged, Navier-Stokes equations in strong conservation form with the Beam and Warming approximate factorization algorithm. The implicit scheme uses central differencing for a curvilinear set of coordinates. The code was originally developed as AIR3D by Pulliam and Steger. ${ }^{11}$ Pulliam ${ }^{12}$ later added the Jameson 13 artificial dissipation and called the code ARC3D. Copper ${ }^{10}$ adapted the ARC3D computer code for internal propulsion applications and named the code PARC3D. The turbulence model used in PARC3D is the Baldwin-Lomax ${ }^{14}$ model which is also an algebric eddy viscosity model. In the present computations; the turbulence model has been modified to improve the simulation of the reverse flow regions based on the study Deiwert. ${ }^{15}$ In the regions of reverse flow, the inner layer is replaced with the with the outer model which extends all the way to the wall. In the absence of reverse flow, the conventional Baldwin-Lomax turbulence model is used. 


\section{RESULTS AND DISCUSSIONS}

The experiment on which this study is based comes from the AGARD Fluid Dynamics Panel Working Group 13 numerical sub-group Test Case 3.16 AGARD Working Group 13 was formed to investigate the subject of inlet performance using results from both experimental and computational techniques. Emphasis was placed on the assessment of inlet performance flow distortion, and the evaluation of relevant distortion parameters over a wide range of inlet flow conditions. One of the test cases chosen by Working Group 13 was the RAE inlet model M2129, which is a circular inlet followed by an S-duct diffuser. The M2129 inlet duct geometry, chosen by AGARD Working Group 13 because of the comprehensive data available, is shown in Fig. 1 and was based on a study by Willmer, Brown, and Goldsmith. ${ }^{17}$ The centerline of the inlet defined in terms of the coordinate system shown in Fig. 1 is given by:

$$
Z_{c l}=-\Delta Z_{c l}\left[1-\cos \left(\pi \frac{X_{c l}}{L}\right)\right]
$$

where $X_{c l}$ is the X-coordinate of the inlet duct centerline, and $\Delta Z_{c l}$ in the centerline offset. The radius distribution measured perpendicular to the duct centerline is given by:

$$
\left(\frac{R-R_{i}}{R_{e f}-R_{i}}\right)=3\left(1-\frac{X_{c l}}{L}\right)^{4}-4\left(1-\frac{X_{c l}}{L}\right)^{3}+1
$$

where $R_{i}$ is the inlet throat radius, $R_{e f}$ is the engine face radius, and $L$ is the length of the inlet. For the purposes of the calculations, the M2129 S-duct was nondimensionalized with respect to the throat radius, thus $R_{i}=1.0, R_{e f}=1.183, L=7.10$, and $\Delta Z_{c l}=2.13$.

A polar grid topology was chosen for the M2129 S-duct which consisted of 49 radial, 49 circumferential, and 61 streamwise nodal points in the half plane, for a total number of 146,461 grid points. The internal grid was constructed such that the transverse computational plane was perpendicular to the duct centerline. Grid clustering was used in the radial direction in order to rudistribute the nodal points to resolve the high shear regions near the wall. The flow in the inlet was considered turbulent throughout. The inflow boundary layer condition corresponds to a shear layer thickness $\delta / R_{i}=0.120$, and were applied one diameter upstream of the inlet entrance in the constant area extension.

A summary of the inlet test conditions used in this study is presented in Table I, and covers the inlet throat Mach number range from 0.1 to 0.794 . It also includes the AGARD Test Case 3.1 an 3.2 test conditions defined by:

\section{Test Case 3.1 Test and Initial Conditions}

Total Pressure

Total Temperature

Throat Mach Number

Throat Diameter

Throat Area

Reynolds Number (based on $D_{i}$ )
$P t_{0}=29.889$ in. $\mathrm{Hg}$

$T t_{0}=293^{\circ} \mathrm{K}$

$M_{i}=0.794$

$D_{i}=5.071$ in.

$A_{i}=25.254$ in. $^{2}$

Rey $=1.848 \times 10^{6}$ 


\section{Test Case 3.2 Test and Initial Conditions}

Total Pressure
Total Temperature
Throat Mach Number
Throat Diameter
Throat Area
Reynolds Number (based on $D_{i}$ )

$$
\begin{aligned}
& P t_{0}=29.865 \mathrm{in} . \mathrm{Hg} \\
& T t_{0}=293^{\circ} \mathrm{K} \\
& M_{i}=0.412 \\
& D_{i}=5.071 \mathrm{in} . \\
& A_{i}=25.254 \mathrm{in.}^{2} \\
& \text { Rey }=1.158 \times 10^{6}
\end{aligned}
$$

For the purposes of examining the the separation characteristics within the M2129 inlet S-duct with both Full Navier-Stokes and Reduce Navier-Stokes solution techniques at a low inlet throat Mach number, a third Test Case was defines as

\section{Test Case 3.3 Test and Initial Conditions}

\author{
Total Pressure \\ Total Temperature \\ Throat Mach Number \\ Throat Diameter \\ Throat Area \\ Reynolds Number (based on $D_{i}$ )
}

$$
\begin{aligned}
& P t_{0}=29.889 \mathrm{in.} \mathrm{Hg} \\
& T t_{0}=293^{\circ} \mathrm{K} \\
& M_{i}=0.200 \\
& D_{i}=5.071 \mathrm{in} . \\
& A_{i}=25.254 \mathrm{in.}^{2} \\
& R e y=0.594 \times 10^{6}
\end{aligned}
$$

Also presented in Table I is the computed location of separation $X_{\text {sep }}$ from both the FNS and RNS analyses (as measured in terms of the arc length along the inlet centerline), the area averaged engine face total pressure recovery $P R$, and the $D C_{60}$ engine face distortion, both determined from the flow values on the computational mesh.

The initial flow field for the Full Navier-Stokes computations was obtained from the Reduced Navier-Stokes (RNS) solution. This was done to reduced the computing time of the Full Navier-Stokes solver. In addition, the imposition of the inlet boundary layer, which was held fixed during the time-marching FNS solution, was straight forward since the initial flow field had the correct initial inlet boundary layer. Examination of saving in the computing time of the Full Navier-Stokes computations using the RNS solution as the initial flow field compared to that using a simple uniform flow field is currently underway.

Figures (1) and (2) present the effect of inlet throat Mach on the area averaged total pressure recovery $P_{t_{e}} / P t_{0}$ and $D C_{60}$ engine face distortion as determined from the Reduced Navier-Stokes and Full Navier-Stokes solution technique. The two solution techniques gave essentially the same total pressure recovery characteristics over the inlet throat Mach number ranged investigated, Fig (2). However, differences between the FNS and RNS solutions were observed when comparing the circumferential distortion as measured in terms of the $D C_{60}$ engine face descriptor, Fig. (3). Both the FNS and RNS analysis indicated that the M2129 inlet separated over the Mach number range from 0.1 to 0.794 . The location of separation, as determined from both the Full Navier-Stokes and Reduced Navier-Stokes solution techniques, is presented in Fig. (4), and suggests that some of the differences in the $D C_{60}$ engine face distortion indicated in Fig. (3) can be attributed to differences in the prediction of flow separation location within the inlet duct. Figure (4) also indicates that the location of flow separation is effected by inlet throat 
Mach number, and that no consistant statement can be made as to the prediction of separation using either FNS or RNS solution techniques. An examination of the predicted separation location for both Full Navier-Stokes and Reduced Navier-Stokes analyses relative to the experimentally measured separation point as well as a comparison of the experimentally measured inlet performance is presently underway.

Presented in Figs. (5) through (8) are a comparison between the predicted engine face total pressure recovery map using both Reduced Navier-Stokes and Full NavierStokes solution techniques at the Test Case $3.1,3.2$, and 3.3 initial flow conditions. In general, there is strong similarity between the engine face flow field as computed by the two Navier-Stokes techniques, although the FNS solution at the Test Case 3.1 and 3.2 flow conditions indicated a somewhat more developed recovery map. This was not true at the Test Case 3.3 initial flow conditions. The more developed engine face flow field computed by the FNS analysis is indicated in Fig. (4) as a higher $D C_{60}$ engine face distortion. At the Test Case 3.3 flow conditions, the underdevelopment of the engine face recovery map of the FNS solution, as compared to the RNS solution, is indicated as a lower $D C_{60}$ distortion.

The nature of the computed differences between the Full Navier-Stokes and Reduced Navier-Stokes solutions technique is clearly indicated in Figs. (8) an (9), which presents the radial pressure ring distortion, Fig. (8), and the $60^{\circ}$ sector circumferential ring distortion characteristics, Fig. (9). The radial pressure ring distortion as computed by both Navier-Stokes techniques is essential equivalent at the Test Case 3.2 initial conditions. This is also true at the Test Case 3.1 and 3.3 initial flow conditions. The primary differences in the prediction of flow separation as computed by Full Navier-Stokes and Reduce Navier-Stokes analyses shows as a difference in circumferential pressure distortion as indicated in Fig. (9) and also Fig. (3). If agreement between solution techniques is measured in terms of standard engine face distortion descriptors, then the two Navier-Stokes analyses gave remarkably similar results for a inlet duct experiencing flow separation characterized as vortex lift-off.

Presented in Figs. (10) through (13) are comparision between the static pressure and wall skin friction distribution along the $\theta=180^{\circ}$ surface element for the Test Case 3.1 initial conditions, Figs. (10) and (11), and at the Test Test Case 3.2 flow conditions, Figs. (12) and (13). The static pressures distributions as determined from the FNS and RNS solution technique are in surprising agreement, Fig. (10) and Fig. (12). The major difference between Full Navier-Stokes and Reduced Navier-Stokes solutions occure in the wall skin friction distribution upstream of flow separation, Figs. (11) and (13). i.e. the region of adverse pressure gradients in the first section of the S-duct. The skin friction in the separated region is characterized as very constant and very close to zero for both analysis techniques. Thus, the near wall velocity distributions within the region were vortex lift-off takes place are very small with very little variation relative to the velocities in the outer region of the flow. The differences in computed skin friction by the two Navier-Stokes solutions within the separated regions are very small.

Figures (14) and (15) present the surface streamline pattern indicating the topology of vortex lift-off as given by the Reduced Navier-Stokes solution technique at the Test Case 3.3 initial condition. A very striking and significant feature captured by the analysis is the convergence of the limiting streamlines as an indication of three dimensional sepa- 
ration taking place in this duct. Another important and striking feature is the symmetric pair of spiral nodes and pair of saddle points that were clearly captured by the 3D RNS analysis.. The topological patterns, as shown in the Figs. (14) and (15), also reveal the remarkable characteristic that the limiting streamlines forming the spiral node enter only from downstream of the nodal point. The very familiar topological pattern is known to describe the important stage in the development of the pair of counter rotating vortices that form in the first section of turning resulting in vortex liftoff in the second section.

\section{CONCLUDING REMARKS}

A three-dimensional implicit Full Navier-Stokes (FNS) analysis and a 3D Reduced Navier Stokes (RNS) initial value space marching solution technique has been applied to a class of separated flow problems within a diffusing S-duct configuration characterized as vortex-liftoff. Both Full Navier-Stokes and Reduced Navier-Stokes solution techniques were able to capture to overall flow physics of vortex lift-off, however more consideration must be given to the development of turbulence models for the prediction of the locations of separation and reattachment. This accounts for some of the discrepancies in the prediction of the relevant inlet distortion descriptors, particularly circumferential distortion. The 3D RNS solution technique using FLARE approximations adequately described the topological and topographical structure of flow separation associated with vortex liftoff. 


\section{REFERENCES}

1 Advisory Group for Aerospace Research and Development (AGARD), "Engine Response to Distorted Inflow Conditions," AGARD CP-400, Sept., 1986.

2 Bowditch, D. N. and Coltrin, R. E., "A Survey of Inlet/Engine Distortion Compatability," AIAA Paper No. 83-1166, June 1983.

${ }^{3}$ Harlof, G. J., Reichert, B. A., and Wellborn, S. R., "Navier-Stokes Analysis and Experimental Data Comparison of Compressible Flow in Diffusing S-Duct," AIAA Paper No. 92-2699, June, 1992.

4 Anderson, B. H. and Farokhi, S.,"A Study of Three Dimensional Turbulent Boundary Layer Separation and Vortex Flow Control Using the Reduced Navier Stokes Equations," Turbulent Shear Flow Symposium, Munich, Germany, Sept. 1991.

5 Briley, W. R. and McDonald, H., "Analysis and Computation of Viscous Subsonic Primary and Secondary Flow," AIAA Paper No. 79-1453.

6 Briley, W. R., and McDonald, H., "Three-Dimensional Viscous Flows with Large Secondary Velocities," Journal of Fluid Mechanics, March 1984, vol. 144, pp. 47-77.

7 Levy, R., Briley, W. R., and McDonald, H., "Viscous Primary/Secondary Flow Analysis for Use with Nonorthogonal Coordinate Systems," AIAA Paper No. 83-0556, Jan. 1983.

8 Anderson, B. H., "The Aerodynamic Characteristics of Vortex Ingestion for the F/A-18 Inlet Duct," AIAA Paper No. 91-0130, Jan. 1991.

9 McDonald, H., and Camarata, F. J.: "An Extended Mixing Length for Computing the Turbulent Boundary-Layer Development, Proceedings, Standford Conference of Turbulent Boundary Layers," Vol. I, Pub. by Standford University, pp 83-98, 1969.

10 Cooper, G. K., "The Parc Codes," Arnold Engineering Development Center, Tullahoma, TN, AEDC-TR-87-24, Oct. 1987.

II Pulliam, T. H., and Steger, J. L., "Implicit Finite-Difference Simulation of ThreeDimensional Compressible Flow," AIAA Journal, Vol. 18, No. 2, 1980, pp 159-167.

12 Pulliam, T. H. "Euler and Thin Navier-Stokes Codes: ARC2D, ARC3D," Notes for Computational Fluid Dynamics User's Workshops, Univ. of Tennessee Space Institute, Tullahoma, TN, UTSI Pub. E02-4005-023-84, 1984, pp 15.1-15.85.

13 Jameson, A., Schmidt, W., and Turkel, E., "Numerical Solutions of the Euler Equations by Finite-Volume Methods Using Runge-Kutta Time-Stepping Schemes," AIAA Paper No. 81-1259, June 1981.

14 Baldwin, B., and Lomax, H. "Thin-Layer Approximation and Algebric Model for Separated Flows,' AIAA Paper No. 78-257, Jan. 1978.

15 Deiwert, G. S., "Computation of Separated Transonic Turbulent Flows," AIAA Journal, Vol. 14, No. 6, June, 1976, pp735-740.

16 Advisory Group for Aerospace Research and Development (AGARD), "Air Intakes for High Speed Vehicles," Fluid Dynamics Panel Working Group 13, AR-270, Sept. 1991. 17 Willmer, A. C., Brown, T. W., and Goldsmith, E. L., "Effects of Intake Geometry on Circular Pitot Intake Performance at Zero and Low Forward Speeds," Aerodynamics of Power Plant Installation, AGARD CP301, Paper No. 5, 1981. 


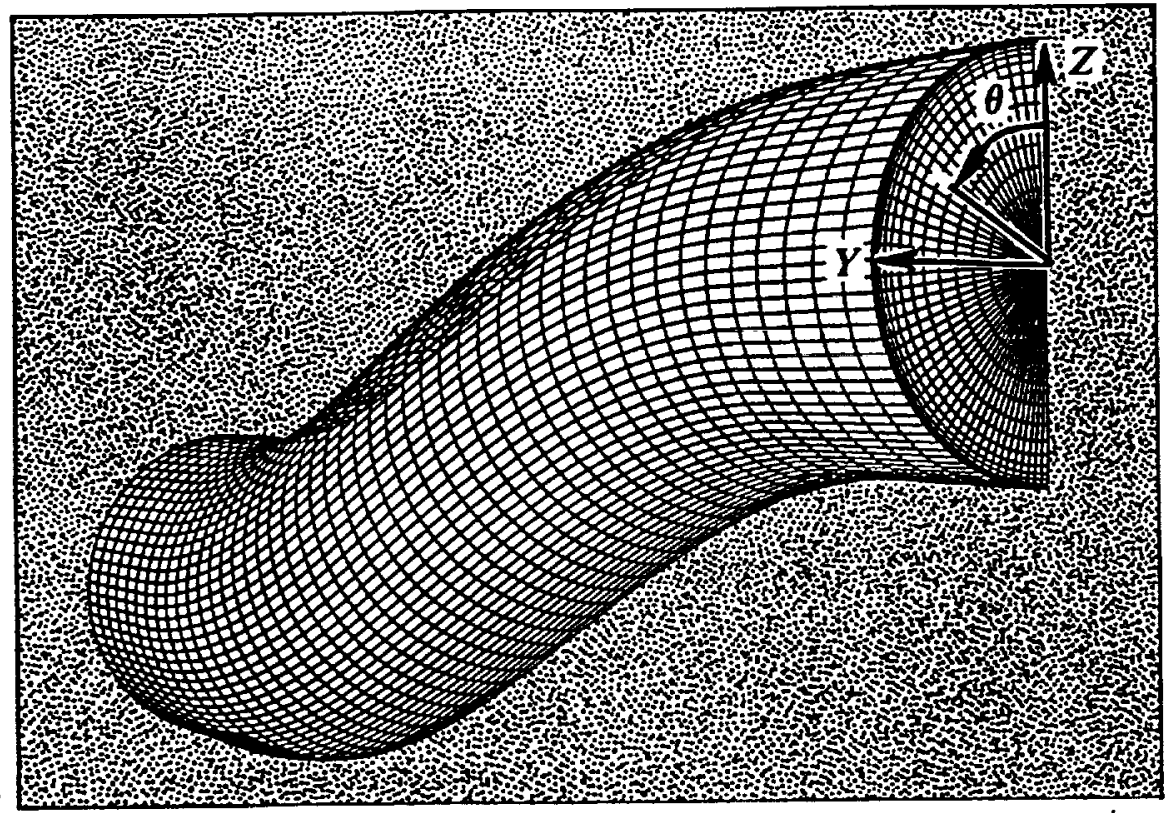

Fig. (1) - Geometry definition for the M2129 intake duct.

\begin{tabular}{|c|c|c|c|c|c|c|c|}
\hline $\begin{array}{l}\text { Mach } \\
\text { No. }\end{array}$ & $\begin{array}{c}\text { Reynolds } \\
\text { No. }\end{array}$ & Xsep & $\begin{array}{c}\text { S Solut } \\
\text { PR }\end{array}$ & DC60 & Xsep & $\begin{array}{l}\text { Solut } \\
\text { PR }\end{array}$ & DC60 \\
\hline $\begin{array}{l}0.100 \\
0.200 \\
0.300 \\
0.412 \\
0.500 \\
0.600 \\
0.700 \\
0.794\end{array}$ & $\begin{array}{l}0.301 \times 10 \mathrm{e} 6 \\
0.594 \times 10 \mathrm{e} 6 \\
0.873 \times 10 \mathrm{e} 6 \\
1.158 \times 10 \mathrm{e} 6 \\
1.385 \times 10 \mathrm{e} 6 \\
1.577 \times 10 \mathrm{e} 6 \\
1.723 \times 10 \mathrm{e} 6 \\
1.848 \times 10 \mathrm{e} 6\end{array}$ & $\begin{array}{l}4.456 \\
4.897 \\
5.109 \\
5.323 \\
5.109 \\
4.675 \\
4.236 \\
3.794\end{array}$ & $\begin{array}{l}0.999 \\
0.997 \\
0.994 \\
0.990 \\
0.985 \\
0.979 \\
0.971 \\
0.962\end{array}$ & $\begin{array}{l}0.320 \\
0.273 \\
0.254 \\
0.254 \\
0.267 \\
0.297 \\
0.348 \\
0.416\end{array}$ & $\begin{array}{c}. .89 \\
4.856 \\
-. .- \\
-.- \\
4.674\end{array}$ & \begin{tabular}{c}
0.996 \\
0.987 \\
\hdashline- \\
$ב .958$
\end{tabular} & $\frac{0.229}{0.321}$ \\
\hline
\end{tabular}

Table I - Initial flow conditions and summary of inlet performance. 


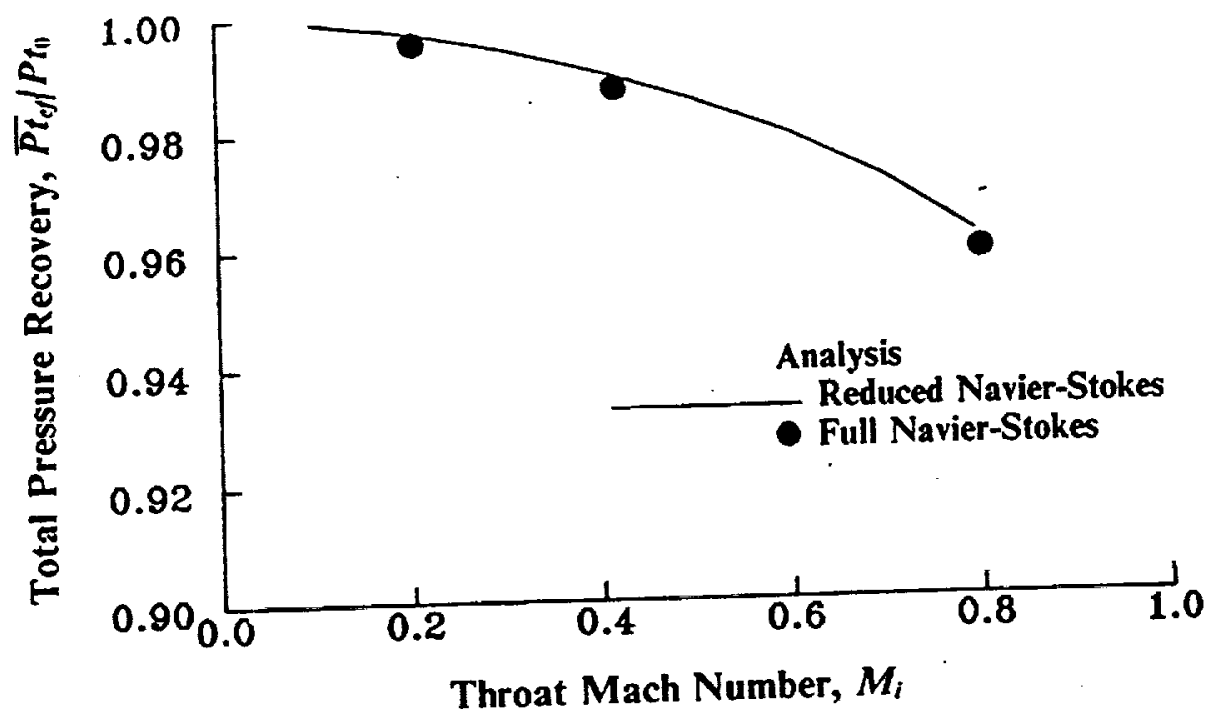

Fig. (2) - Effect of inlet throat Mach number on engine face total pressure recovery for the M2129 intake duct.,

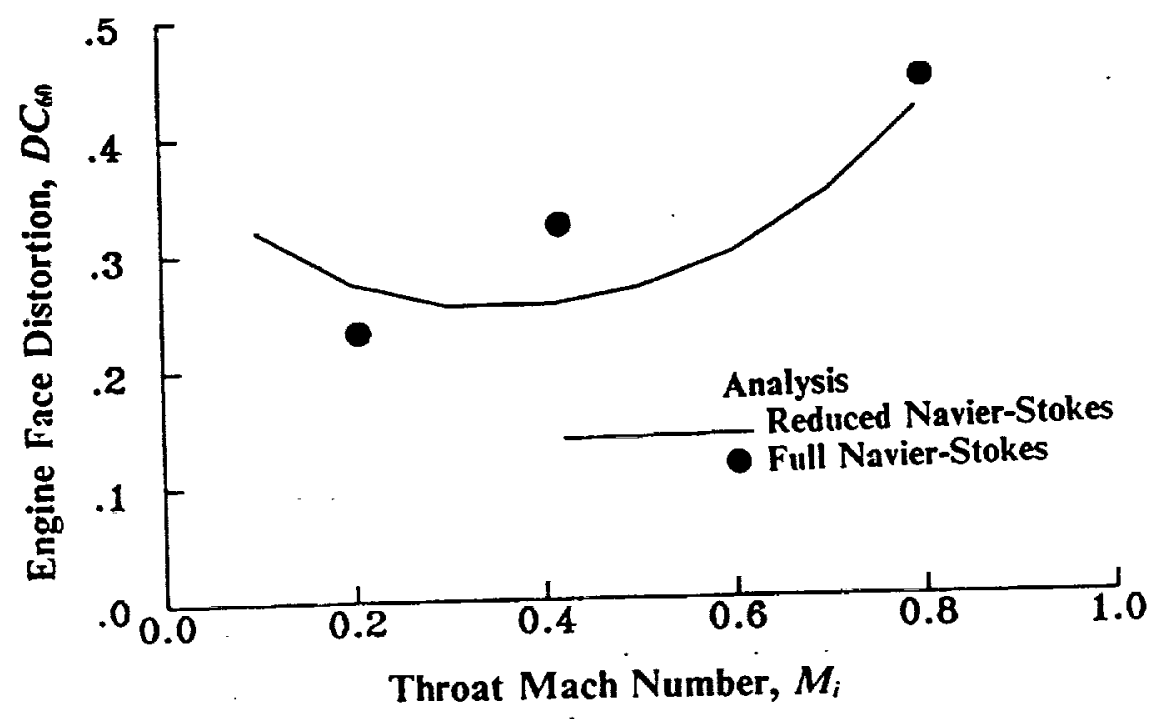

Fig. (3) - Effect of inlet throat Mach number on $D C_{60}$ engine face distortion for the M2129 intake duct. 


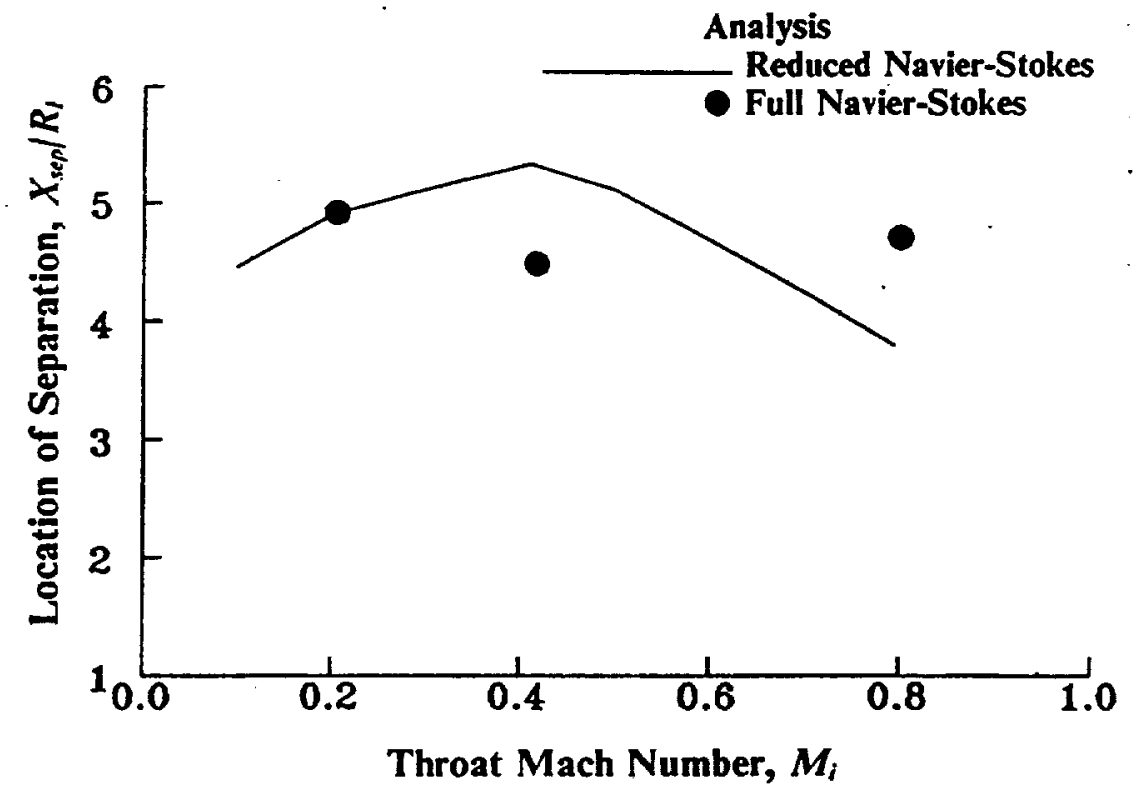

Fig. (4) - Effect of inlet throat Mach number on the location of flow separation in the M2129 intake duct.

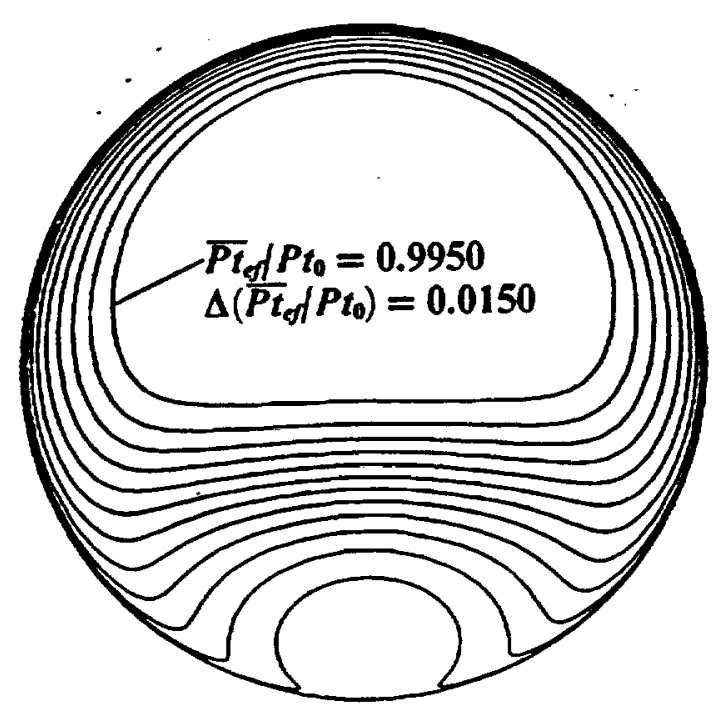

RNS ANALYSIS

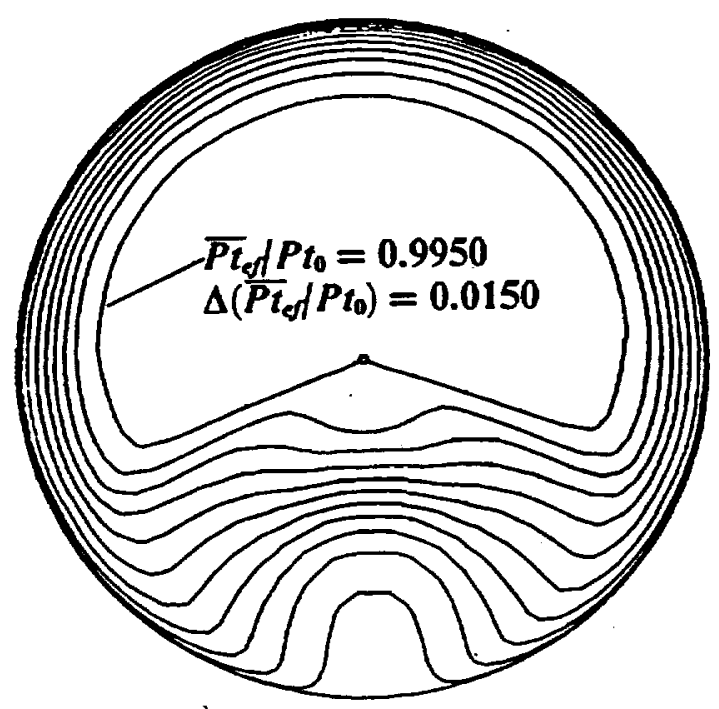

FNS ANALYSIS

Fig. (5) - Engine face flow field for the M2129 intake duct, Test Case 3.1 initial conditions. 


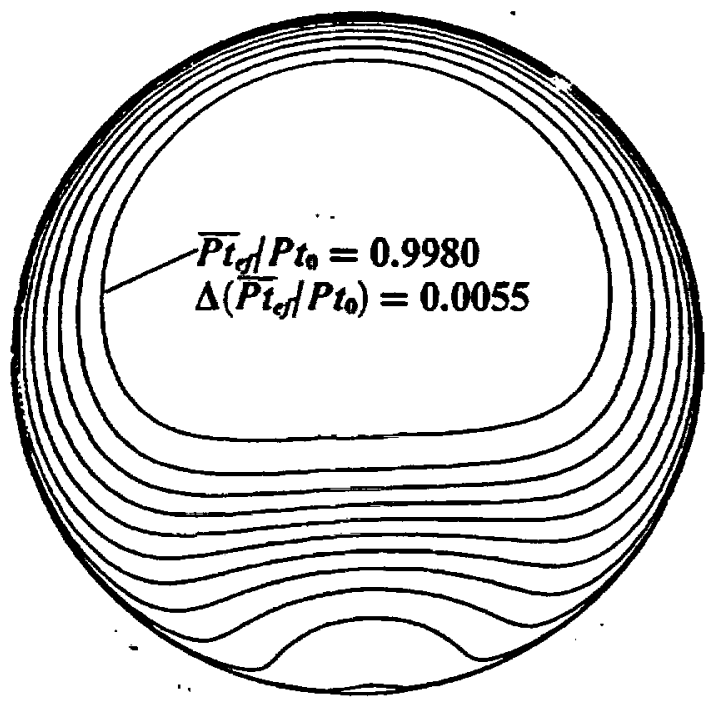

RNS ANALYSIS

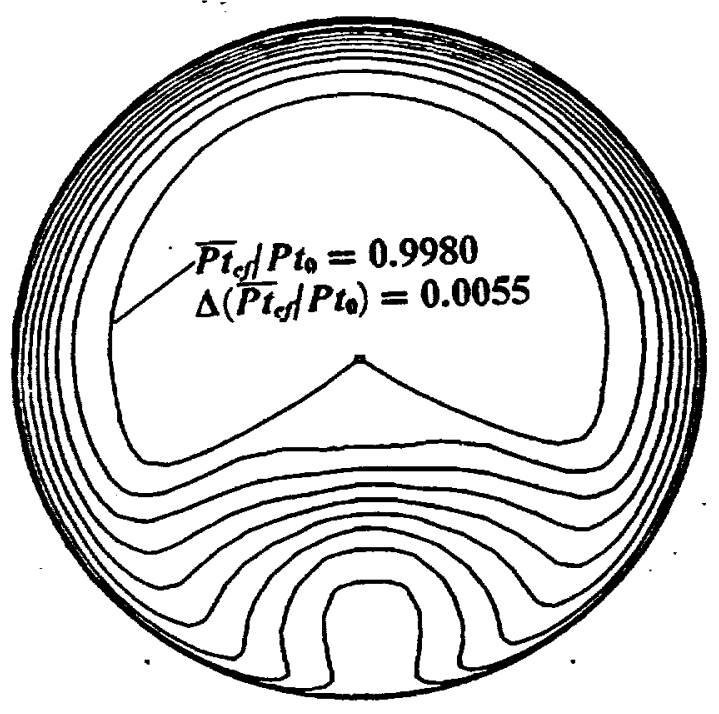

FNS ANALYSIS

Fig. (6) - Engine face flow field for the M2129 intake duct, Test Case 3.2 initial conditions.

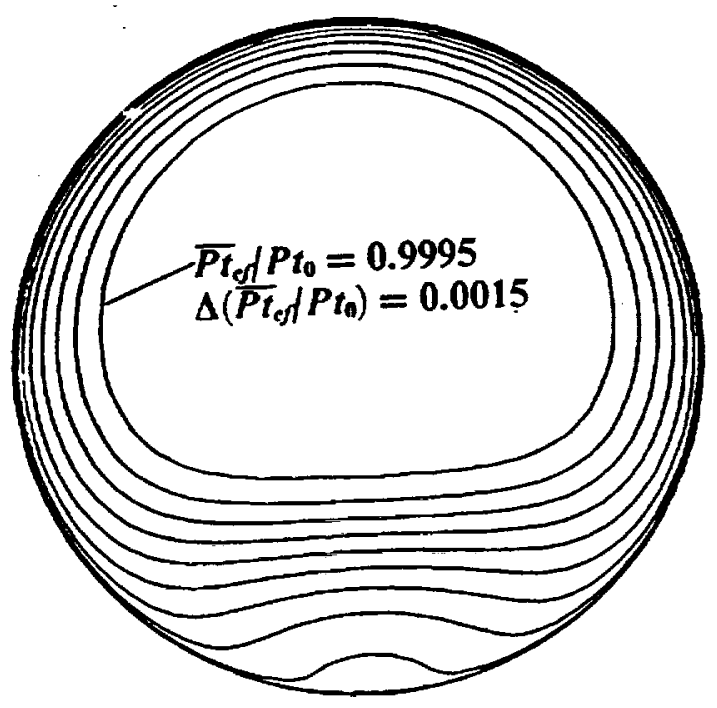

RNS ANALYSIS

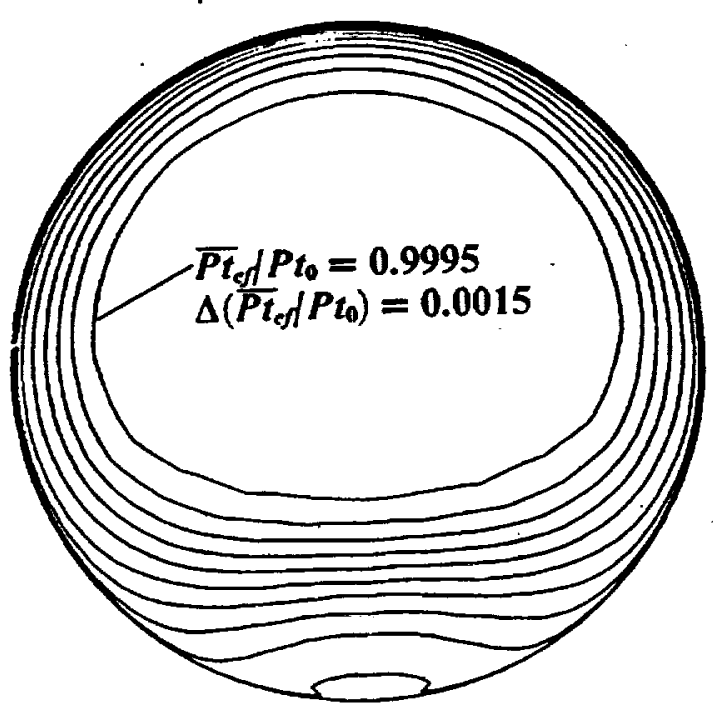

FNS ANALYSIS

Fig. (7) - Engine face flow field for the M2129 intake duct, Test Case 3.3 initial conditions. 


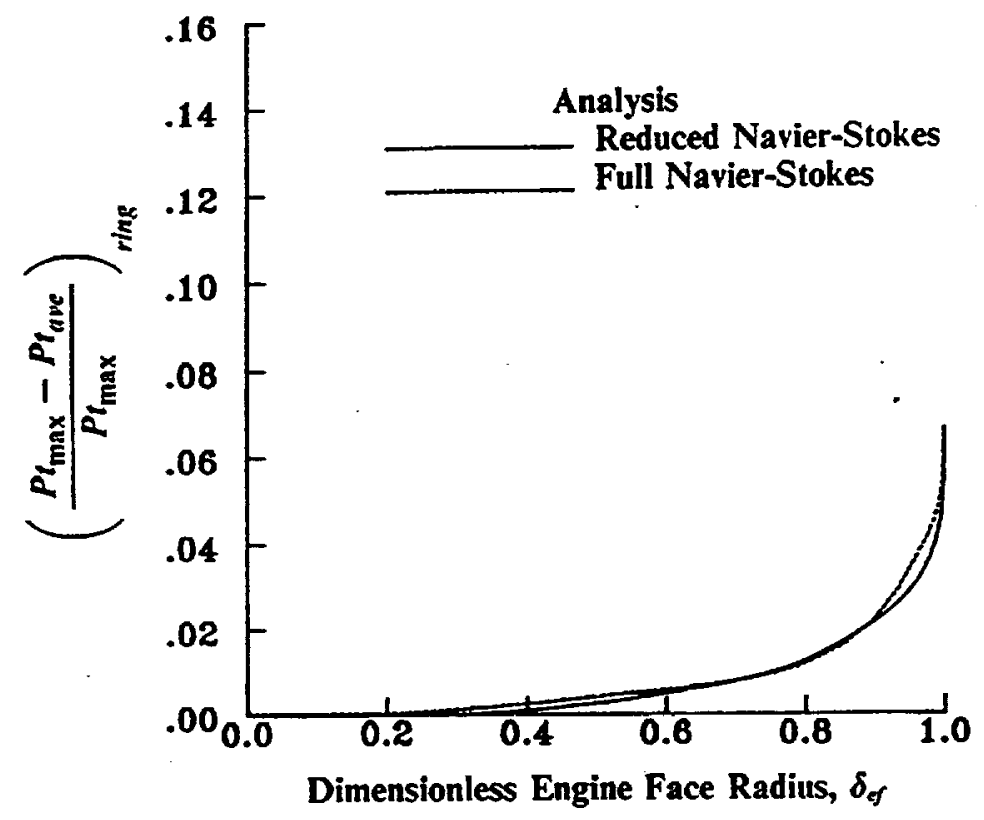

Fig. (8) - Radial pressure ring distortion characteristics, Test Case 3.2 initial conditions.

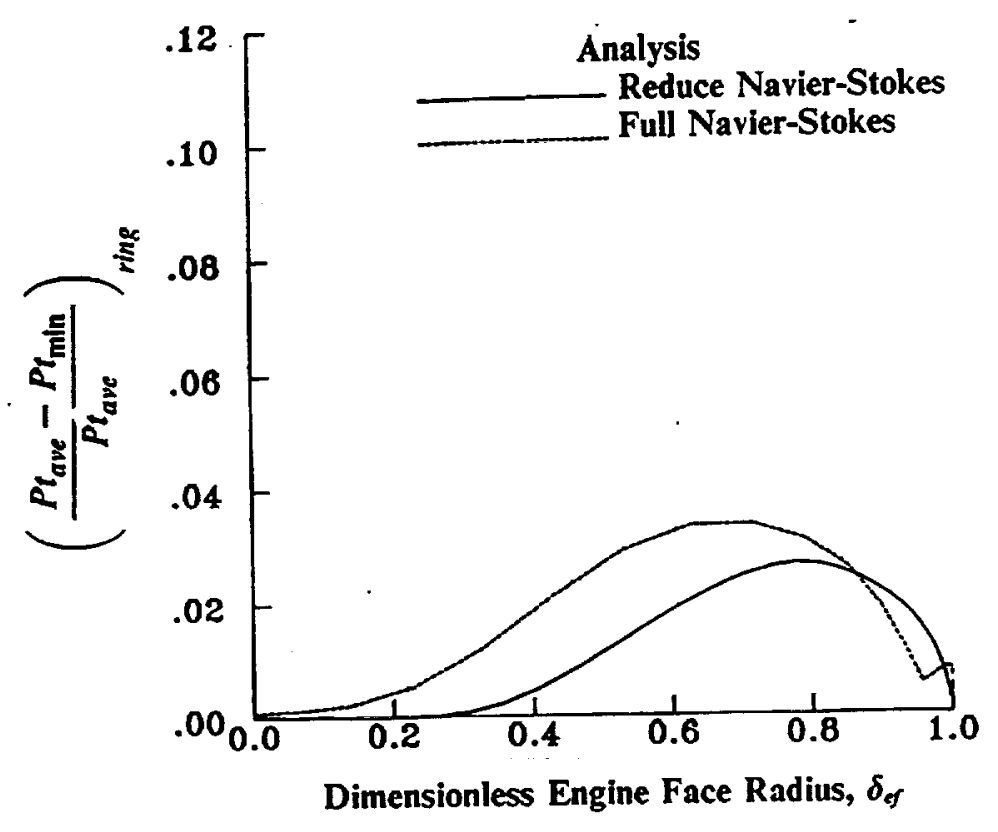

Fig. (9) - $60^{\circ}$ - sector circumferential pressure ring distortion characteristics, Test Case 3.2 initial conditions. 


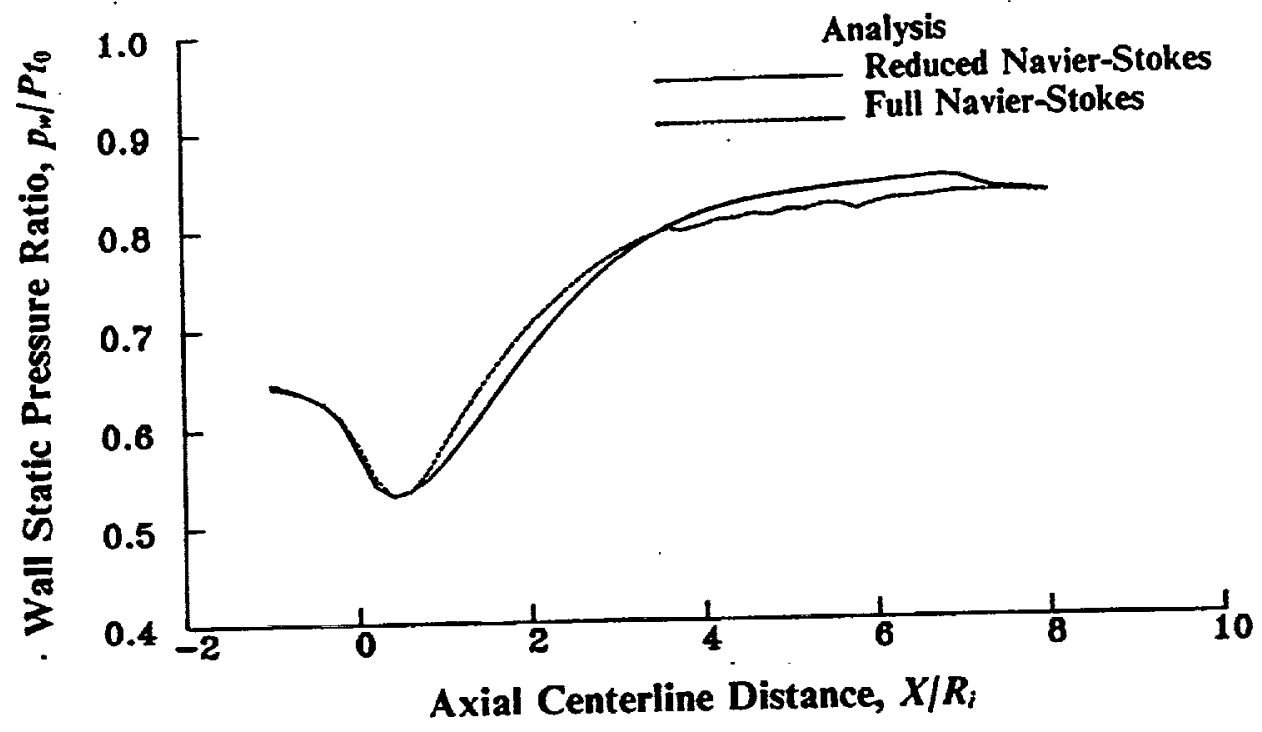

Fig. (10) - Static pressure distribution along the $\theta=180^{\circ}$ surface element, Test Case 3.1 initial conditions.

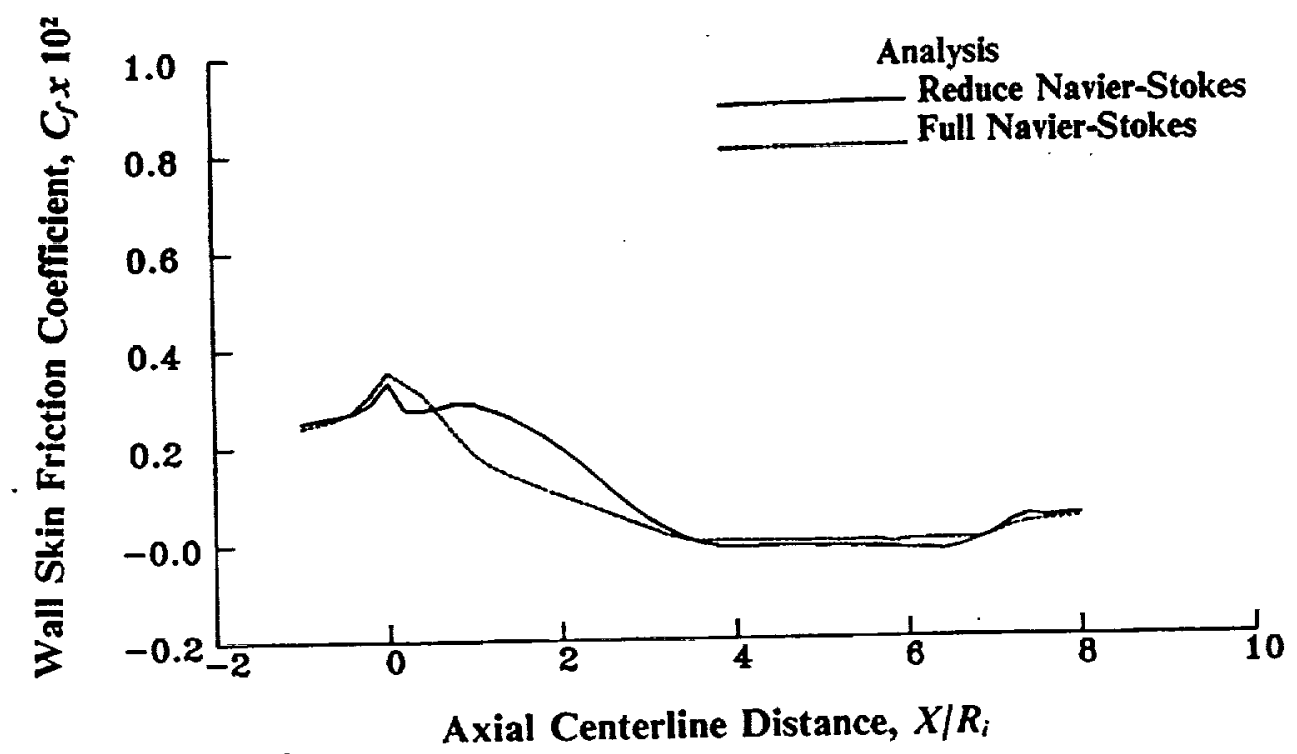

Fig. (11) - Wall skin friction distribution along the $\theta=180^{\circ}$ surface element, Test Case 3.1 initial conditions. 


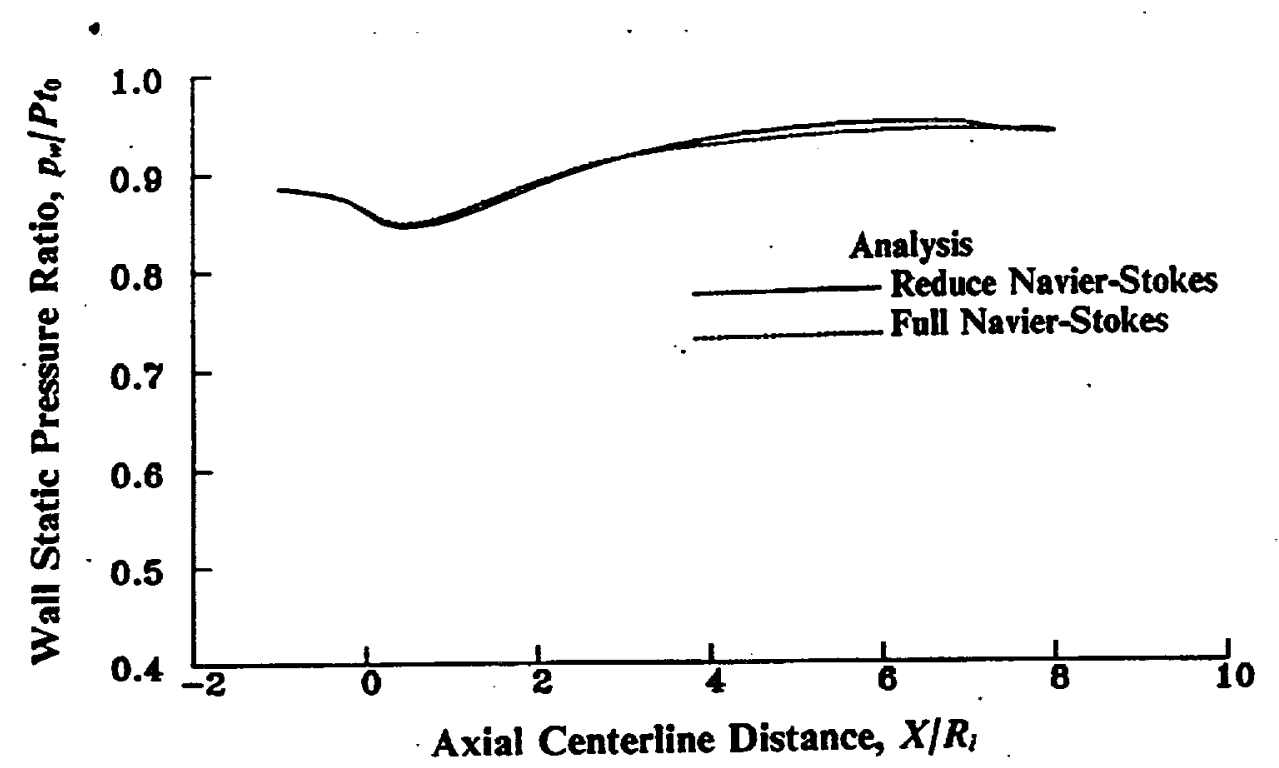

Fig. (12) - Static pressure distribution along the $\theta=180^{\circ}$ surface element, Test Case 3.2 initial conditions.

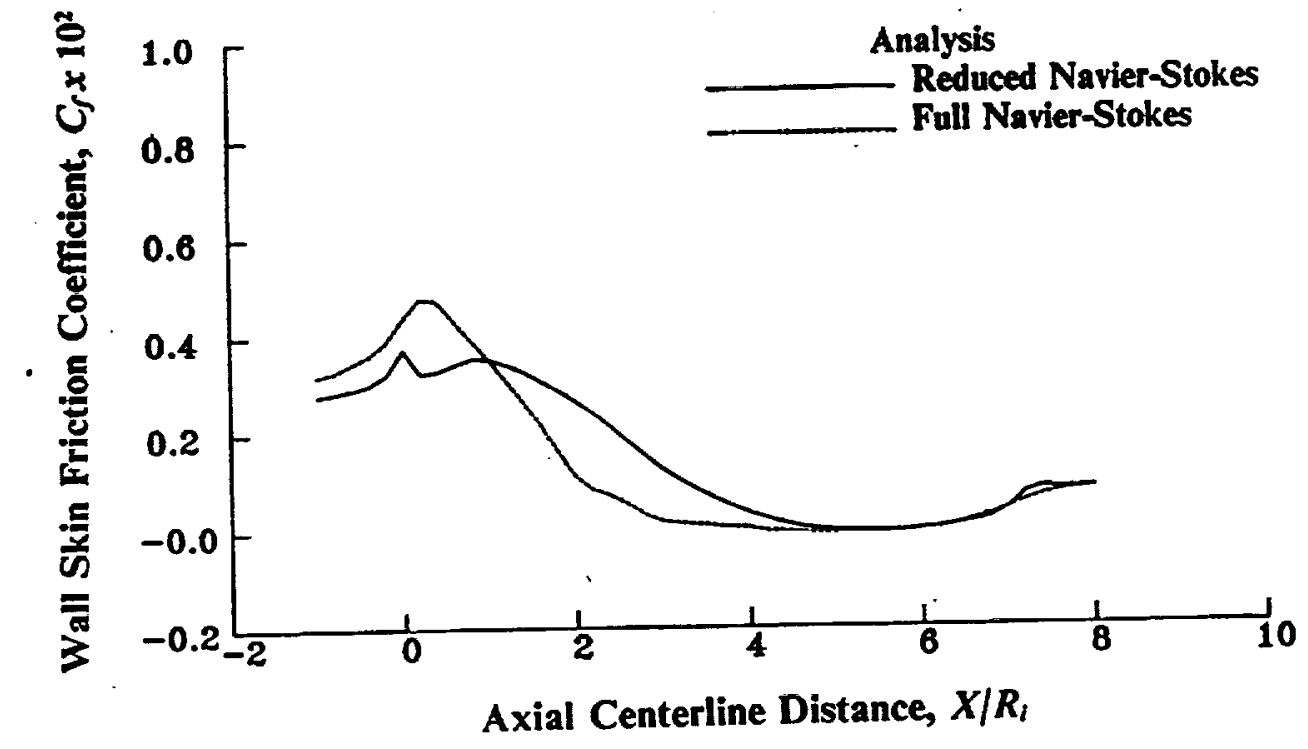

Fig. (13) - Wall skin friction distribution along the $\theta=180^{\circ}$ surface element, Test Case 3.2 initial conditions. 


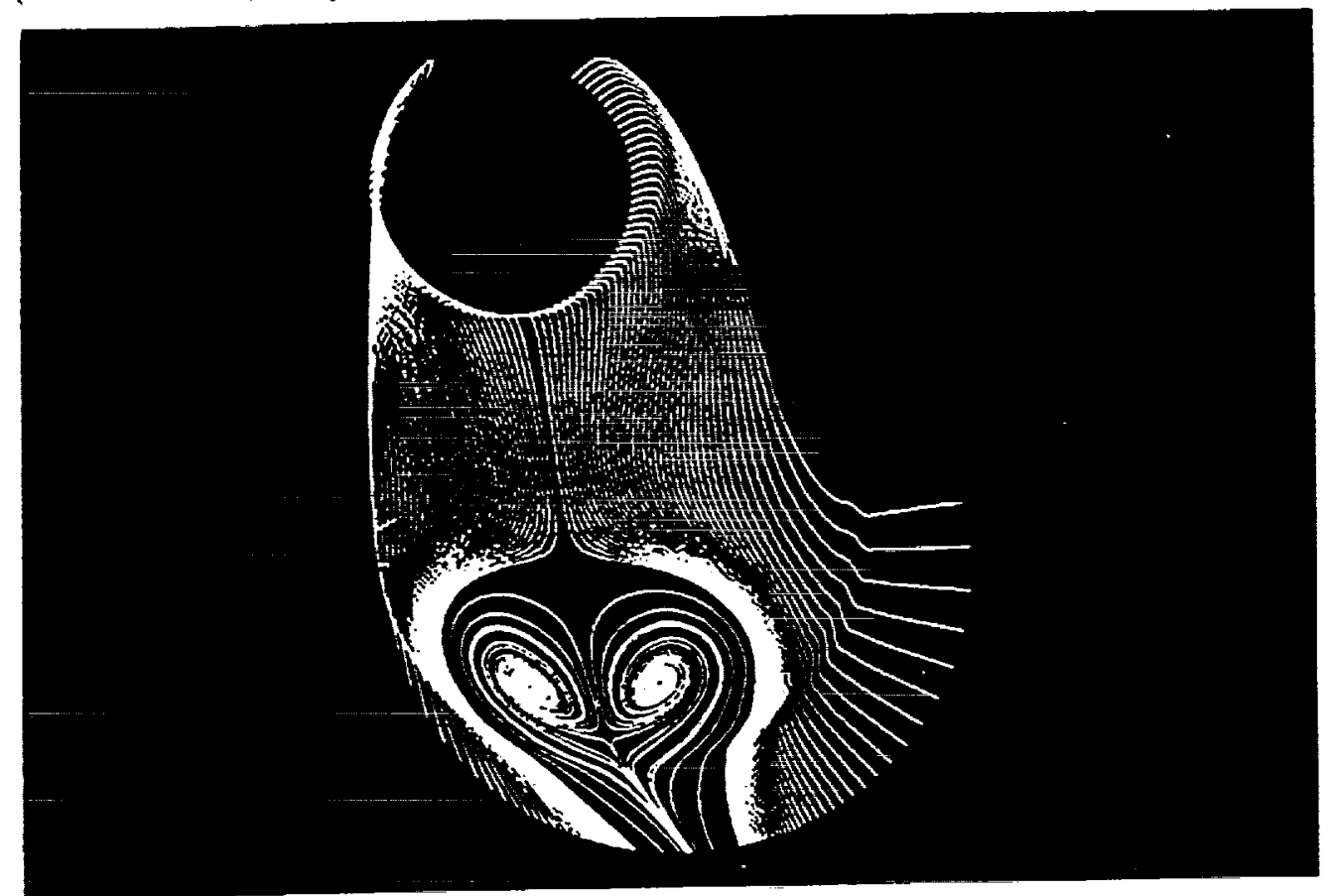

Fig. (14) - Surface streamlines pattern showing the topology of vortex liftoff, Reduced Navier-Stokes solution, Test Case 3.2 initial conditions.

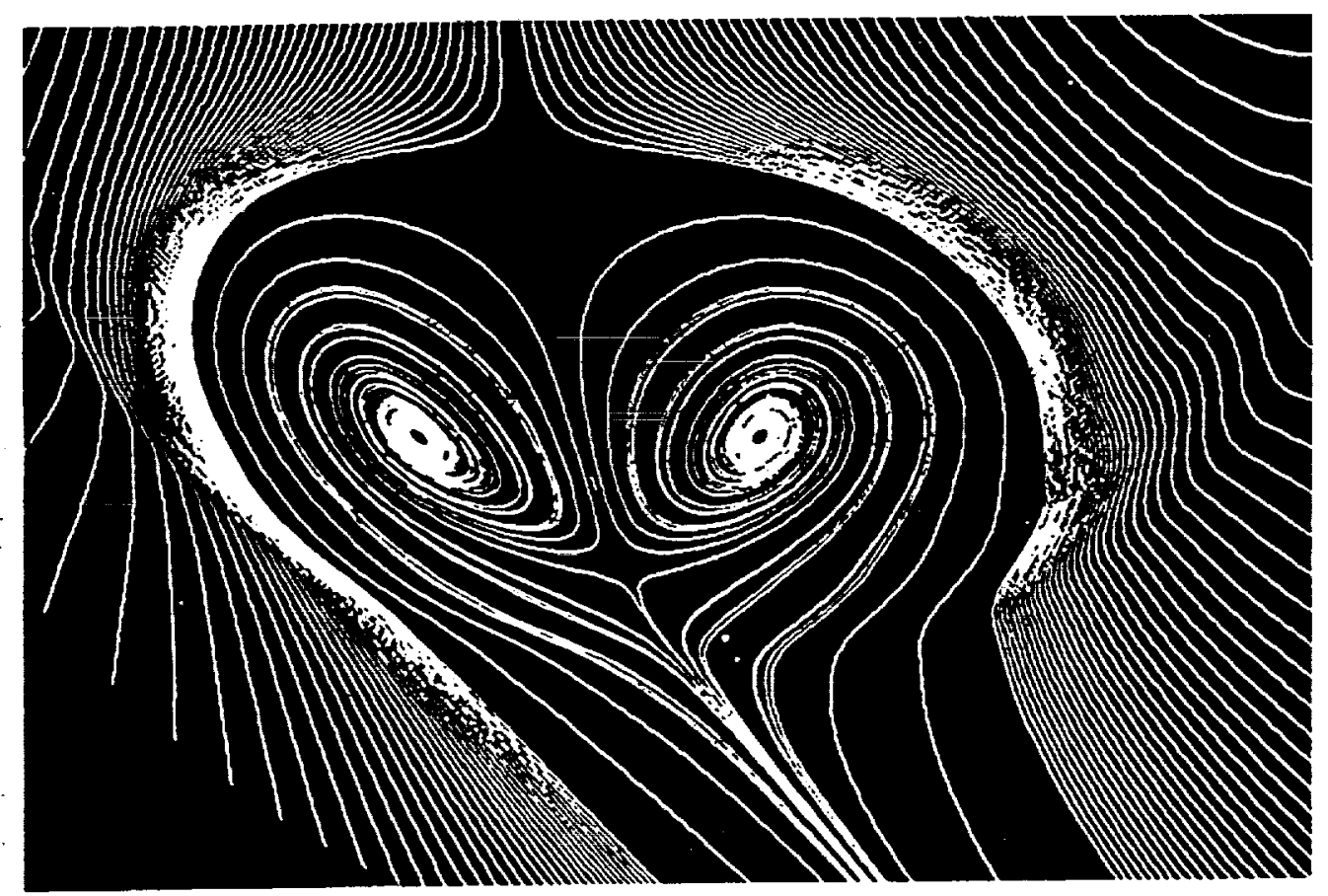

Fig. (15) - Enlarged streamline pattem showing the topology of vortex liftoff, Reduced Navier-Stokes solution, Test Case 3.2 initial conditions. 



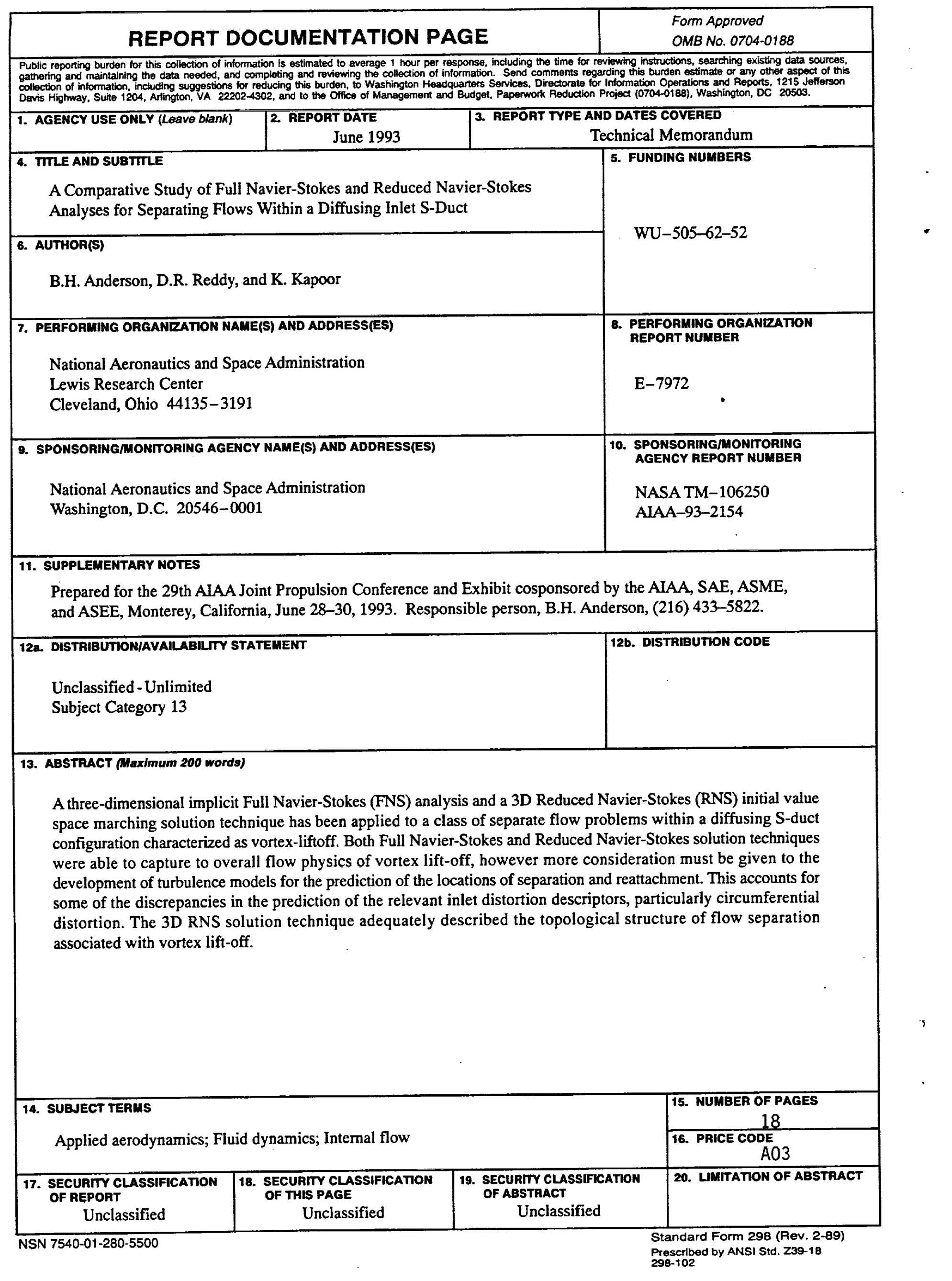

